\title{
Incorporating quality control activities in scrum in relation to the concept of test backlog
}

\author{
MUHAMMAD AAMIR and MUHAMMAD NAEEM AHMED KHAN* \\ Shaheed Zulfikar Ali Bhutto Institute of Science and Technology (SZABIST), Islamabad 44000, Pakistan \\ e-mail: amir_amirkhan94@yahoo.com; mnak2010@gmail.com
}

MS received 18 June 2015; revised 29 October 2016; accepted 27 January 2017

\begin{abstract}
Scrum has become popular among the agile methodologies due to the substantial support that it provides to the project management teams. The scrum process delivers quick functionality in the form of sprints. Most of the quality assurance and quality control activities are skipped in scrum because of its short lifecycle and due to the absence of a dedicated quality assurance team. The development team pays more attention to delivery of products according to the customer satisfaction, and the parameters used for this type of assessment are the story success criteria and user acceptance testing. Only acceptance testing and integration testing are not sufficient to achieve a quality product. There is still a clamouring need felt to incorporate other quality control activities in scrum to achieve a quality product. In this work, we have made an attempt to implement the quality control activities in the scrum philosophy by introducing the concept of test backlog. The enhanced scrum model provides quality assessment methodology on the basis of frequency of remaining functional bugs. The concept of test backlog proposed in this study aims at implementing state of the art testing process in scrum. A case study was carried out to validate the model, which produced satisfactory results. Besides, we conducted a survey to access the state of the quality control in scrum. The proposed model and case study results are reported herein.
\end{abstract}

Keywords. Rapid application development; agile software development; extreme programming; scrum; software quality assurance.

\section{Introduction}

Quality control (QC) activities, especially testing, are a vital part of every software project. Software development is a complex task and several constraints are taken into consideration to achieve it. The main constraints that require proper planning and management are cost, scope and time to achieve a quality product. Scrum is a lightweight software development process framework and is widely used in the industry due to its short development lifecycle and quick response to change requests. Scrum is neither a development method nor a process; rather, it is a framework with which we can employ various processes and techniques to address complex problems [1]. Scrum is relatively simple to understand and considerably difficult to master. The scrum framework consists of scrum team, roles and responsibilities of team members, events, artefacts and specific rules that bind all these components together [2]. The scrum process delivers the functionality quickly in the form of a product increment. Each increment contains some business functionality so that project can be completed within its stipulated timeframe. The team size in scrum is generally small-a maximum of nine persons. Further,

*For correspondence time-consuming activities such as large documentation are avoided, which reduce development efforts and product cost.

Scrum is based on empirical process control theory. The theory is based on three pillars: transparency, inspection and adaption. Transparency means that the employed process and its outcome should be visible to all the participants [3]. Inspection means that all the participants' actions/activities must be inspected by an inspector; typically, the inspectors are called scrum masters. Adaption means that the suggestions and guidelines solicited by the inspector must be adopted. The start-of-the-art scrum approach suggests focussing on quick delivery with abstract level testing (i.e., go live decision is purely based on story success criteria). We suggest performing start-of-the-art testing activities in scrum to deliver quality work. Test backlog in scrum is a new concept and we suggest using it to sustain test cases.

Scrum framework consists of the following four main components:
a. scrum team,
b. team roles,
c. artefacts,
d. events. 
a. Scrum team: The scrum team includes product owner, development team and scrum master. Schwaber and Sutherland [1] describe that scrum teams are self-organizing and cross-functional. The scrum team develops the product iteratively and delivers it incrementally, which maximizes the feedback opportunities.

b. Team roles: The scrum teams have the following roles and responsibilities:

i. Product backlog is an artefact that contains the user stories. Product owner role includes managing these stories such that the stories are understandable to the development team and scrum master. These user stories work as a requirement engineering technique for scrum [4]. The product owner must have sufficient knowledge about the client's organization and business. On the basis of this knowledge, the product owner prioritizes the product backlog items. Anyone who wants to change the product backlog items should convince the product owner beforehand.

ii. Development team consists of professional developers and sometimes testers are also included, who actually develop the deliverables and verify it according to the story success criteria. Development team members are self-organizing and cross-functional. Development team does not contain dedicated team for testing or business analysis [3]. The size of the team ranges from three to nine people. The scrum master and product owner are not included in this count.

iii. Scrum master is the expeditor and acts as a coach for the scrum team. Scrum master provides services to the product owner and development team [2]. Scrum master helps product owner in finding techniques for effective product backlog management and maintains slope of the scrum process. Scrum master coaches the organization in adoption of scrum, and generates awareness among the stakeholders that changes in the requirements increase the productivity of scrum. The overall success of scrum is based on the effective leading strategies practiced by the scrum master.

c. Artefacts: Scrum artefacts represent the work in a proper way to maximize transparency of the scrum process [4]. Scrum framework contains two types of artefacts: product backlog and sprint backlog.

i. Product backlog contains all the requirements of the software product. The requirements contained in the product backlog are generally in the form of user stories [5]. Each story is three to five lines long. The items are contained in the backlog in a prioritized form. The product owner is responsible for ordering and maintaining the backlog items $[2,4]$. The product backlog constantly keeps changing because of the dynamic nature of software requirements.

ii. Sprint backlog contains a set of product backlog items for a particular sprint plus a plan for delivering the increment [6]. Once the sprint starts, the contents of the sprint backlog are locked down. In case a change in the sprint backlog is required, it will take place by the permission of the product owner and when the contents are modified then the product owner will restart the sprint. The normal duration for a sprint ranges from two to four weeks.

There are two other types of artefacts that are used to forecast progress of the scrum. These artefacts are known as burndown-charts and definition-of-done. The burndownchart is a projective practice used to forecast the sprint progress. The definition-of-done describes the conditions that assess whether the work is complete on the product increment. It is imperative that all the participants must understand the meaning of "done".

d. Events: The scrum framework contains a number of events and each event is a formal opportunity to inspect and adopt something [5]. Sprint is a container of all the events. The main events in scrum are the sprint, the sprint planning meeting, daily scrum meeting, sprint review meeting and sprint retrospective.

i. The sprint: Sutherland and Schwaber [6] describe that sprint is a heart of scrum. The sprint is an event during which a working code is developed, and at the end of sprint the done increment is delivered. It is not necessary that each sprint should be delivered; sometimes more than one increments are integrated and delivered to the client. The delivery of sprints depends on the nature of project and customer needs. Once a sprint is completed, the new sprint starts immediately. Afterwards, all the remaining events such as sprint planning meeting, sprint review meeting, daily scrum and sprint retrospective are carried out in the sprint event. A sprint can be cancelled before time and the authority to cancel the sprint rests with the product owner.

ii. The sprint planning meeting: The sprint planning meeting involves all the scrum team members. The work to be done is planned in the sprint planning meeting. The sprint planning meeting is generally $8 \mathrm{~h}$ meeting for a 1 month sprint.

iv. The sprint review meeting: The sprint review meeting is held at the end of each sprint. During the sprint review, all the stakeholders and the scrum team members ponder upon what was done in the current sprint [5]. The duration of this meeting is usually $4 \mathrm{~h}$ for a 1 month sprint. At this meeting, an assessment is also made on the knowledge gained through a particular sprint [7]. 
v. The sprint retrospective meeting: The sprint retrospective meeting is held after the sprint review meeting and before the next sprint planning meeting. At this meeting, quality of the work done is evaluated and an assessment of the whole team is carried out [8].

Scrum is widely used for complex software development. Time, cost and scope are the three main factors that articulate success and effectiveness of a framework. Scope defines the features and functions of the product. In particular, scope means functional requirements. QC activities are performed to verify the product before its release and identify deviations from the requirements (in the form of bugs). The scrum framework uses an incremental approach for software development that minimizes the development time and cost. Mostly, quality is not taken into consideration in the scrum framework due to the quick delivery process of sprints. Scrum team delivers some part of the software functionality in a short time and verifies the deliverables on the basis of "definition-of-done" or story success criteria. Due to this, sometimes team members overlook quality of software product and as a result end up with a pile of quality-related items [9]. Further, a software developer cannot write a bug-free code when working under pressure. Scrum advocates for short development cycles. It has been observed that the QC activities are not carried out in scrum that affect the deliverables as well as the final product quality. Since the system is developed and delivered to the customers in the form of small increments, assessing quality of such small increments itself becomes a key challenge. The following two research questions have been identified and are answered in the fourth section.

Research question 1: How can we incorporate the QC activities in scrum?

Research question 2: How to assess quality of the product being developed?

This paper consists of seven sections. In the first section, we describe characteristics of the scrum framework. In the next section, we discuss research background and related work carried out on quality issues in scrum. This section also discusses key reasons of QC activities in scrum. In the third section, we propose an enhanced quality-focussed model of scrum in which we account for the test backlog. The model validation through a case study carried out in a middle level software company is discussed in the fourth section. We also conducted a survey about our research. The survey results are provided in the fifth and sixth sections. Finally, this paper concludes with a summary and we identify some possible future dimensions to this research in the last section.

\section{Related work}

Due to rapidly changing business demands in the modern era, large organizations and companies operating within the ambient of information-intensive industries are increasingly adopting agile/scrum to promptly modify IT functionality [10]. After carrying out meticulous investigation of the issues associated with scrum adoption, Paulk [11] states that better comprehension of the perceived benefits and worth of scrum and its leverage points might encourage scrum adoption and may lead to its widespread and effective deployment in the IT industry. To highlight the importance of quality assurance (QA) involvement in the scrum process, Larusdottir et al [2] performed an in-depth survey to highlight the effectiveness of usability testing within the purview of scrum by interviewing scrum experts and practitioners. The survey results indicate that the more focussed aspects in scrum are based on its simplicity and speedy development. According to the findings based on the survey results, $72 \%$ of scrum practitioners agreed that QC activities should be engaged throughout the development of software products and $44 \%$ of the survey participants agreed that extensive testing of the software improves the overall product quality. The survey results also indicate that $64 \%$ of practitioners agreed that the testing should be performed at the end of each sprint. Hence, testing in scrum would be an easy task if one of the team members plays role of a software tester.

Löffler et al [4] suggested a testing model to carry out acceptance testing in scrum. The proposed technique employed a model-based technique for documenting the software requirements. The proposed model could be used by the software engineers to elicit requirement specifications and software testers can use it to perform software testing tasks. A key significance of the model is that it stresses upon testing each sprint before its delivery to the customers/clients. The authors employed UML's lightweight modelling notations for writing user stories. The model amalgamates the principles of agile manifesto, model-based testing and agile modelling techniques.

Green et al [3] investigated the relationship between asynchronous and synchronous communication in the domain of agile software development and its overall effects on the software quality. Agile software development sticks to the concepts and principles of colocated teams. Synchronous communication among the colocated teams adds value to ultimate product quality. By translating synchronous communication needs into an appropriate level of asynchronous communication, the agile software development teams working at various locations can achieve a similar success.

Stettina and Hörz [12] assessed the efficacy of employing agile project management approaches in IT project portfolios and outlined the implications and characteristics of agile portfolio management practices in the corporate 
sector and organizations. To deal with complex and multifaceted requirements in an agile development environment, Vlaanderen et al [7] propositioned an "agile requirements refinery," which in fact is an extension of the scrum process that facilitates product managers. The increased deployment of agile approaches (such as scrum methodology) in software development industry has led to the inclusion of agile software development practices in software Engineering curricula at undergraduate level. To boost computer science students' exposure to scrum, Rodriguez et al [13] proposed Virtual Scrum, which is also known as an "educational virtual world" and simulates a scrum-based team room using various virtual elements such as web browser, charts, calendars, blackboards and document viewers. To emphasize the significance of adopting scrum as an agile project management method, von Wangenheim et al [14] developed a paper and pencil game to educate students of undergraduate degree programmes about the benefits and merits of using scrum.

Scrum can be further enhanced by applying SDLC phases [5]. For example, testing phase can be made part of an enhanced scrum process. There are also some distinct QA phases that are helpful to check quality of the product and processes. It is recommended that testers should work side by side with the development teams and each subtask should be tested once it is completed. QA process must commence when the sprint starts, and testing activities should be executed on a daily basis.

A survey was conducted by Akif and Majeed [9] in which all the participants were skilled and experienced scrum practitioners. The survey results led to discovery and highlighting of various issues and challenges in scrum. These challenges include code quality, quality items pileup, module integration, sprint duration, etc. Hongying and Cheng [15] proposed an agile QA model to be used by small-sized and medium-sized QA teams. The proposed agile QA model consists of 20 key process areas and provides guidelines for implementation and customization. Each process area provides pertinent guidelines for agile software development teams to analyse and improve their QA activities.

A longitudinal study was carried out by $\mathrm{Li}$ et al [16] to compare scrum with traditional software development practices and methodologies with particular reference to the QA processes and software defects. The study reports that the software defects density provided by scrum is not only at par with the traditional software development methodologies but also the Scrum attaches more prominence on working of the development teams in terms of short-term time span for product increments. Bhasin [17] ascertained several challenges encountered in agile methodology while assuring software product's quality. The study suggested a framework to assess those aspects of agile methodologies that help enhance the product quality. The proposed framework provides policies and guidelines that could be followed to increase quality of the software products.
Parvez and Parvaz [18] discussed gaps and disparities between the software development team and QA team during the scrum process and proposed an approach to augment communication between both the teams. The proposed process design entails a number of micro-cycles. The number of cycles is in fact proportional to the number of sprints. Each cycle consists of four different phases including planning, development, release and delivery phases.

A process model based on the scrum framework called DT@Scrum is presented by Häger et al [19] to integrate design thinking into the agile software development methodology. In DT@Scrum, the software developers select their individual operation mode after each sprint. Such selections are primarily based on how well the product requirements are understood. To improve success rate of the software projects and enhance the business adaptability for the changing business environment, Tomanek et al [20] emphasized on combining the principles and processes of project management framework and scrum practices with particular emphasis on their use in web development projects. To identify correct business and candidate requirements, requirements prioritization is an effective way. Though prioritization is not an easy or static process in scrum because of ever-evolving requirements, the same is not the case with the traditional development methodologies [21]. Two vital research issues, project monitoring and knowledge sharing, related to integrating Web 2.0 technologies with the scrum methodology are highlighted by Glória et al [22]. The extent to which Web 2.0 technologies help improve performance of scrum teams is also determined in the study.

Usman et al [23] explored how knowledge areas of project management can be embedded into modern software development lifecycle of scrum, eXtreme Programming and RUP to improve software product quality and develop software products at economical costs without time overrun. Stålhane et al [24] assessed the IEC61508 standard to develop a good understanding of the application of scrum to IEC61508 certifiable software production, and based on the study assessments, the authors proposed the Safe Scrum approach, where the principal idea is separation of concerns. The study suggest that everything that is not part of software development process is kept outside scrum as, in the Safe Scrum, all the requirements are split into safety critical requirements and rest of the requirements are inserted into separate product backlogs.

\subsection{Motivation}

To assess the current state of the QC activities in scrum, particularly in relation to the concept of test backlog, we conducted a survey in which participants were the experienced IT professionals working in companies where scrum is being used as a process model. The survey results were 
subsequently used to validate our proposed framework. The survey results highlighted the importance of adaptation and management of QC activities in scrum and, hence, served as a motivation to undertake this research.

\section{Proposed model}

In this section, we provide our model to ascertain QC in scrum. Our proposed model primarily answers the two research questions as described in the first section. The answers to these research questions are provided below.

\subsection{Performing $Q C$ activities in scrum}

To answer this question, we propose an inclusive model (figure 1), which describes a novel approach to perform QC activities in scrum. The concept of test backlog is introduced in this model. The model describes QC activities in a systematic way.

In our proposed model, the $\mathrm{QC}$ activities start in the same way as a sprint starts. It is recommended that QC team should not be part of the scrum team, but quality work team would work side by side with the scrum team. QC team should be involved in sprint review, daily scrum and sprint retrospective events. QC team should perform all QC activities to achieve the final product quality. It has been observed that testing performed by the professional testers generally produces better results as compared with the testing performed by the developers.

Generally, during the software development process, development team has a good problem solving approach and is aware of the actual flow of the software and steps to be performed in accordance with some standard or best practices; this is the reason why the development

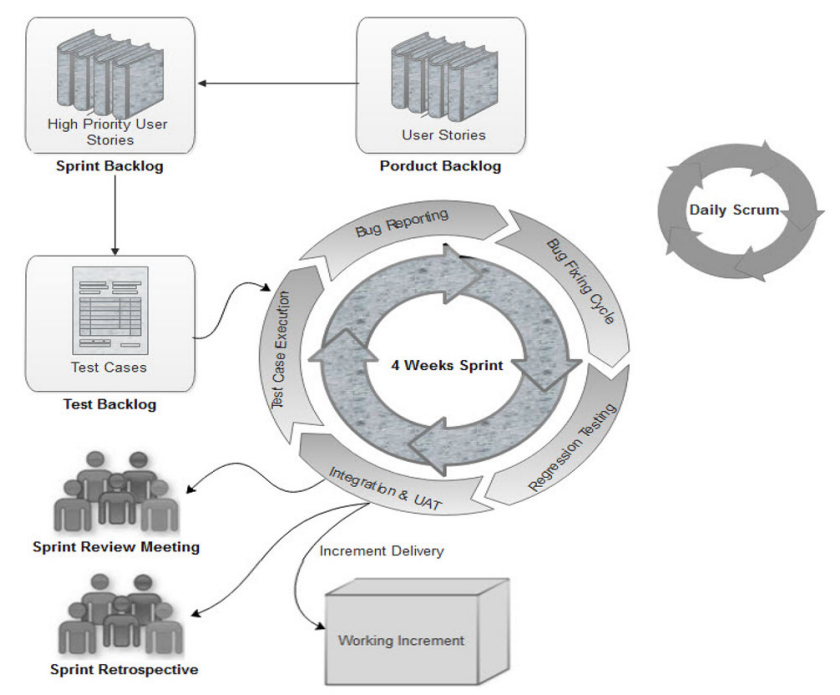

Figure 1. An enhanced scrum model. team cannot easily fail the system when performing system testing. On the other hand, testers have critical thinking and always try to fail the system; for this very reason, they can identify the defects quickly. QC team plays a vital role in the product quality. Therefore, all the quality gates should be part of the "definition-of-done". A brief description of the test backlogs, QC activities in scrum, sprint and scrum ceremonies employed in our model are discussed here.

\subsection{Test backlog}

Test backlog contains the test cases. It has been observed that documented testing is more efficient than exploratory testing. Hence, it is much important to document all the test cases and execute when testing process starts. Test cases are created on the basis of user stories contained in the sprint backlog. Like sprint backlog, test backlog contains test cases in a prioritized form. Test cases should be prioritized on the basis of risks, functionality and frequency of usage. Test cases and user stories should have one-to-many relation, i.e., for a single user story there may be multiple test cases. The number of test cases may increase according to complexity of the user story.

\subsection{QC activities and tester roles in scrum}

QC activities include test case writing, test case execution, system testing, integration testing and regression testing. In our model, we assign utmost preference to the testing process, and other QA activities may be skipped here. A brief description of the $\mathrm{QC}$ activities along with tester roles used in our model is provided here.

a. Test case writing: All the test cases should be written by the QC team/testers and should be placed in the test backlog. Test cases in the test backlog should be prioritized against user stories.

b. Test case execution: Test cases should be executed by testers for each sprint and bugs should be reported accordingly. Test case execution activity will be performed against the functionalities developed and delivered for QA purposes. Testing team members should execute all the test cases on the basis of the priority set in the test backlog. It would be better if we can automate more and more test cases.

c. Unit testing: Unit testing should be performed by the developers. However, testers should guide them about the precise way to perform unit test of a user story. The best way for this discussion is to benefit from daily stand-up meetings.

d. System testing: System testing should be performed only by the testers.

e. Integration testing: Both the development and QC teams should be involved in the integration testing. Module 
integration should be performed by the developers and subsequently verified by the QC team/testers.

f. Regression testing: Once the bugs are fixed, regression testing should be performed by the testers. If the test cases are passed, then the increment is verified and becomes ready to deliver.

If we are running short of time, then the minor bugs that do not affect the system functionality can be ignored. Such bugs should be fixed in the next iteration. We know that each increment delivers some functionality to the client; therefore, the increment should never contain functional bugs.

\subsection{Scrum ceremonies and QC team}

3.4a Sprint planning: QC team should be involved in the sprint planning meeting so that it can understand the required functionality to be developed for a particular sprint. The QC team should prepare test cases according to the functional requirements as well as on the basis of user stories selected for the sprint. The QC team will be in overall charge and responsible for providing estimations about data creation, generating/preparing test cases and writing test scripts, acceptance of test design, executing the test cases, scripting tasks, setting up the environments for the test run, etc. The QC team also defines acceptance criteria for the user stories for the next sprint based on certain QA perspectives such as standards and possible performance issues. When the team feedback is received, the acceptance criterion is updated accordingly and corresponding test cases are created as part of the requirements for acceptance testing.

3.4b Daily scrum: In the daily scrum meeting, the testers should be involved in discussing the functional bugs count identified since the last meeting and how many of them are verified and fixed. Testers can better guide the developers in identifying unit test cases for a given user story. Testers can communicate with developers to declare what is "definition-of-done" for the given user story. Further, testers can also explain why the already delivered user story is not being tested yet. In this meeting, developers can discuss which defects are currently being fixed and which are delayed as well as the reasons behind yet unfixed defects.

3.4c Sprint retrospective: In sprint retrospective meeting, the QC team should provide necessary suggestions and guidelines to improve quality of the product and way and means through which the current process can further be improved.

Now we address the second research question mentioned in the first section.

\subsection{Assessing quality of the product being developed}

The software development team develops the software functionality and the QC team tests that functionality.
Continuous integration and continuous testing are key to the quality of the product being developed. As discussed earlier, functional bugs affect quality of the product and sometimes they may delay the product delivery. Bugs tracking tools should be used to report and track the bugs. To assess quality of the product being developed, we take into account three parameters: number of functional bugs, bugs frequency (or remaining bugs/defects) and time to deliver. The remaining bugs can be calculated by subtracting the fixed bugs from the total bugs:

$$
\text { remaining bugs }=\text { number of total bugs }- \text { fixed bugs. }
$$

3.5a Assumptions: Here we make two assumptions. The first assumption is that if the frequency of bugs shows upwards trend then it means that the development team does not have the ability to resolve the bugs in a timely manner as required in scrum. Hence, we can assume here that the quality of product is going down. A bugs frequency chart is shown in figure 2 . It shows that quality of the product goes down if frequency of remaining bugs is high.

The second assumption corresponds to the view that if the frequency of bugs goes down with the passage of time, then it means that quality of the product goes up. A graph is created on the basis of two parameters, which are frequency of remaining bugs and time. Like the burndown-chart, the quality graph (figure 3 ) provides a closer picture of product quality. The $x$-axis and $y$-axis show time (in days) and frequency of bugs, respectively.

\section{Case study}

To validate our model, we carried out a case study of a software development project spanning over 4 months in a medium-sized software developing company. The main theme of the project was to develop a digital asset management system. We distributed the project into four increments. The modular compatibility and dependability were calculated and on the basis of these calculations, distributions of the modules and tasks were carried out. The whole system was developed in four sprints. Each sprint was 4 weeks long and contained approximately 100 tasks. Higher priority tasks were developed in the first cycle. The development team consisted of one scrum master, one product owner, five developers and one QC engineer. The role of QC engineer was to manage testing backlog, design and execute test cases as well as prepare test reports and verify the identified bugs. The test cases were written on the basis of user stories identified in each sprint. It is worth mentioning that the number of test cases was more than the number of user stories. Test automation improves the testing process and we adopted this approach in our study. Most of the testing tasks were carried out through the automated scripts specifically developed for this purpose. The statistics of the first sprint are given in table 1 . 
Bugs

\section{Bugs Frequency}

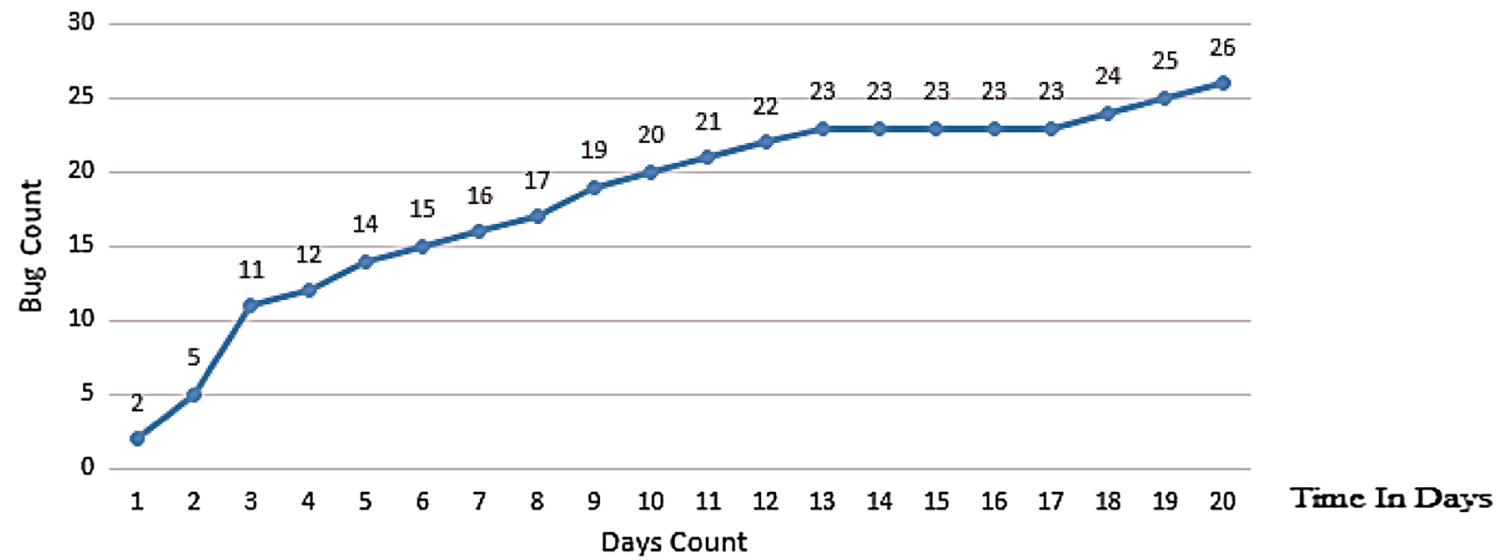

Figure 2. Bugs Frequency Chart-I (indicating that quality of the product goes down if frequency of remaining bugs is high).

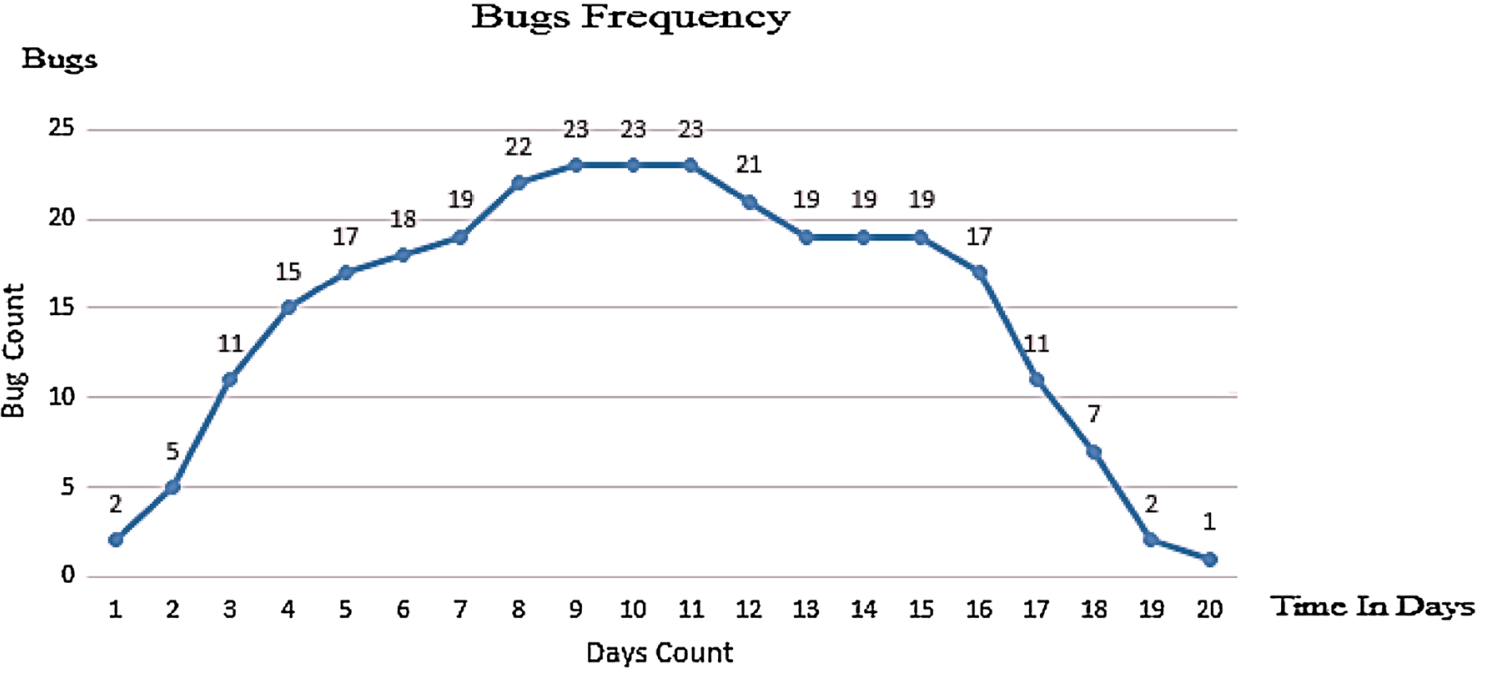

Figure 3. Bugs Frequency Chart-II (indicating that quality of the product goes up if frequency of remaining bugs is small).

In the first sprint, we did not get satisfactory results because members of the development team were naive to work in the agile environment. However, with the passage of time, the team became familiar with the agile technique. Therefore, in the next three sprints, we started getting more satisfactory results in terms of bugs prevention and control, quick development and team collaboration. The quality was improved up to $56 \%$ on the basis of frequency of remaining bugs. The most critical bugs were given special considerations and small bugs were deferred and moved into the next sprint to resolve them later. This approach also helped produce $50 \%$ satisfactory results than the other projects that were being developed in the company. The statistics of the second sprint are given in table 2. It is clear from Sprint 2 statistics that the given sprint is much more managed that Sprint 1 in terms of bugs frequency and control.
As shown in table 2, the bug count increased in the middle of Sprint 2, but alongside, the control on bug resolution is also remarkable. Due to this, we have only five open bugs at the end of Sprint 2, which are moved to next sprint. Since we use bug frequency as a parameter for QC, the number of bug resolutions has been assigned priority over the defect prevention measures. On the very first day for the first sprint, we did not have any open bug because that was the first day of development and the functionality was delivered to the QA team at the end of the first day. However, on the first day of the second sprint we had two defects. These defects are identified during the regression testing of the first sprint. The result of the second sprint is much more managed than the first one. During the start of the second sprint, we focussed mainly on the development of features rather than fixing the old bugs. Because of this 
Table 1. Statistics of first sprint.

\begin{tabular}{rrrrrrr}
\hline $\begin{array}{l}\text { Working } \\
\text { days }\end{array}$ & $\begin{array}{c}\text { Ideal } \\
\text { flow }\end{array}$ & $\begin{array}{c}\text { Tasks } \\
\text { per } \\
\text { day }\end{array}$ & $\begin{array}{c}\text { Actual } \\
\text { flow }\end{array}$ & $\begin{array}{c}\text { Total } \\
\text { bugs }\end{array}$ & $\begin{array}{c}\text { Bugs } \\
\text { fixed }\end{array}$ & $\begin{array}{c}\text { Bugs } \\
\text { frequency }\end{array}$ \\
\hline 1 & 100 & 5 & 95 & 5 & 0 & 5 \\
2 & 95 & 2 & 93 & 20 & 15 & 5 \\
3 & 90 & 1 & 89 & 25 & 10 & 15 \\
4 & 85 & 3 & 82 & 19 & 12 & 7 \\
5 & 80 & 3 & 77 & 11 & 1 & 10 \\
6 & 75 & 6 & 69 & 23 & 3 & 20 \\
7 & 70 & 10 & 60 & 16 & 0 & 16 \\
8 & 65 & 8 & 57 & 20 & 7 & 13 \\
9 & 60 & 2 & 58 & 26 & 5 & 21 \\
10 & 55 & 3 & 52 & 30 & 2 & 28 \\
11 & 50 & 8 & 42 & 35 & 7 & 28 \\
12 & 45 & 6 & 39 & 25 & 5 & 20 \\
13 & 40 & 2 & 38 & 18 & 6 & 12 \\
14 & 35 & 15 & 20 & 34 & 21 & 13 \\
15 & 30 & 7 & 23 & 30 & 23 & 7 \\
16 & 25 & 2 & 23 & 26 & 20 & 6 \\
17 & 20 & 7 & 13 & 28 & 27 & 1 \\
18 & 15 & 5 & 10 & 3 & 2 & 1 \\
19 & 10 & 2 & 8 & 2 & 2 & 0 \\
20 & 5 & 1 & 0 & 0 & 0 & 0 \\
\hline & & & & & &
\end{tabular}

exercise, the number of functional bugs kept on increasing day by day. In the middle of the sprint, we noticed that the quality of the application was going down as the functional bugs were constantly increasing. Then the development team started fixing these defects in parallel, and the results clearly commensurate to this effect that we were left with only five minor defects at the end of sprint. After thoroughly investigating the impact and severity of defects, it was decided to declare those defects as open issues and would be fixed in the next sprint.

The proposed model provided us necessary support for project tracking and quality assessment. Further, a systematic approach was introduced to carry out the QC activities in the scrum. The case study results indicate that by adopting the proposed approach, quality products can be developed within the time, cost and scope constraints.

\section{Survey results}

To further validate our framework, we conducted a survey to analyse state of the QC activities in scrum being practiced in the software industry. Statistics were collected and analysed to access three important aspects of QC in scrum including adaptation and management, assessing scrum and scrum enhancement activities. For this purpose, we prepared an elaborate questionnaire pertaining to various aspects of QC. We conducted a survey in which scrum practitioners from the software
Table 2. Statistics of second sprint.

\begin{tabular}{ccccccc}
\hline $\begin{array}{l}\text { Working } \\
\text { days }\end{array}$ & $\begin{array}{c}\text { Ideal } \\
\text { flow }\end{array}$ & $\begin{array}{c}\text { Tasks } \\
\text { per } \\
\text { day }\end{array}$ & $\begin{array}{c}\text { Actual } \\
\text { flow }\end{array}$ & $\begin{array}{c}\text { Total } \\
\text { bugs }\end{array}$ & $\begin{array}{c}\text { Bugs } \\
\text { fixed }\end{array}$ & $\begin{array}{c}\text { Bugs } \\
\text { frequency }\end{array}$ \\
\hline 1 & 80 & 3 & 77 & 3 & 2 & 1 \\
2 & 77 & 6 & 71 & 9 & 7 & 2 \\
3 & 71 & 7 & 64 & 19 & 6 & 13 \\
4 & 64 & 4 & 60 & 17 & 3 & 14 \\
5 & 60 & 5 & 55 & 25 & 22 & 3 \\
6 & 55 & 7 & 48 & 41 & 33 & 8 \\
7 & 48 & 2 & 46 & 18 & 0 & 18 \\
8 & 46 & 5 & 41 & 52 & 21 & 31 \\
9 & 41 & 6 & 35 & 59 & 31 & 28 \\
10 & 35 & 4 & 31 & 51 & 16 & 35 \\
11 & 31 & 3 & 28 & 41 & 16 & 25 \\
12 & 28 & 4 & 24 & 33 & 6 & 27 \\
13 & 24 & 3 & 21 & 51 & 13 & 38 \\
14 & 21 & 3 & 18 & 39 & 11 & 28 \\
15 & 18 & 3 & 15 & 26 & 16 & 10 \\
16 & 15 & 4 & 11 & 39 & 17 & 22 \\
17 & 11 & 3 & 8 & 22 & 17 & 5 \\
18 & 8 & 4 & 4 & 19 & 16 & 3 \\
19 & 4 & 2 & 2 & 12 & 8 & 4 \\
20 & 0 & 2 & 0 & 7 & 2 & 5 \\
\hline & & & & & & \\
\hline
\end{tabular}

industry participated. The survey questionnaire also consisted of some elementary questions like relating and assessing the quality of the software product based on the number of functional bugs. However, for brevity, the results of such survey questions are skipped as their answers were obvious. The survey results are reported in this section and a comprehensive analysis of the survey results is provided in the next section.

\subsection{Adoption and management}

Q1. The survey results for the question "Is Scrum implemented in your organization?" are shown in figure 4.

Q2. The survey results for the question "Scrum is lightweight easy to understand but difficult to manage" are provided in figure 5 .

\subsection{Assessing scrum for QC activities}

Q1. The survey results for the question "Scrum is an agile methodology in which state of the art quality control activities are not fully performed" are shown in the form of a line graph in figure 6.

Q2. The survey results for the question "Is it sufficient to assess the quality of the sprint through story success criteria and definition-of-done only" are provided in the form of a line graph in figure 7. 


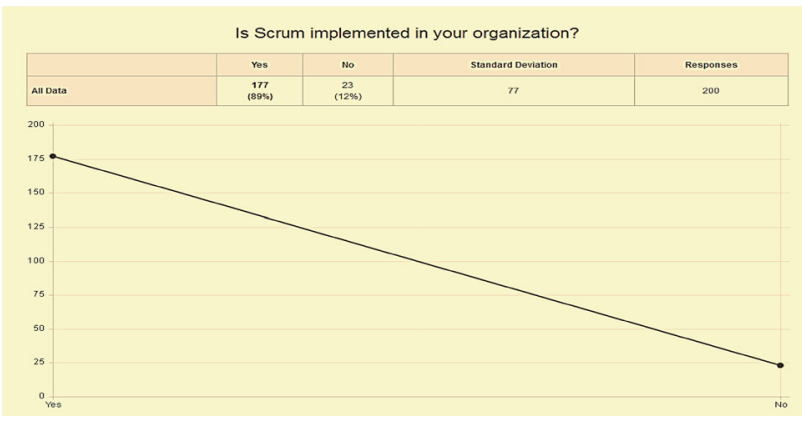

Figure 4. State of scrum implementation in software industry.

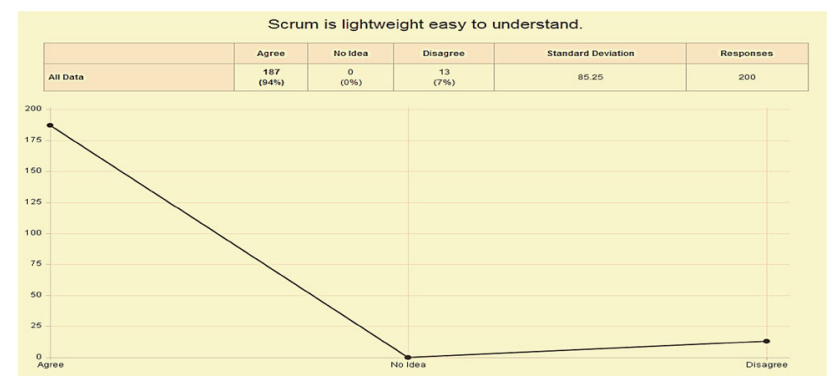

Figure 5. Managing scrum.

\subsection{Proposed QC activites for scrum enhancment}

Q1. The survey results for the questions "Documented testing is a good approach to achieve final product quality" and "It is a good approach to enhance the current Scrum technique by incorporating state of the art quality control activities in the scrum" are shown in figures 8 and 9 , respectively.

Q2. The survey results for the question "It is a good approach to incorporate a Test Backlog in the Scrum to document the test cases" are specified in figure 10 .

Q3. The survey results for the question "It will be good if the QC team work with the Scrum Team in Parallel" are presented in figure 11.

Q4. The survey results for the question "Scrum is difficult to manage" are presented in figure 12 .

\section{Result analysis}

In this section, analyses of the results derived from survey are presented. In all, 200 experienced IT professionals, working in software companies where scrum is used as a process model, participated in the survey. All the professionals who participated in the survey had more than 10 years of working experience in their respective domains. Besides 51 scrum masters, there were 38 product owners

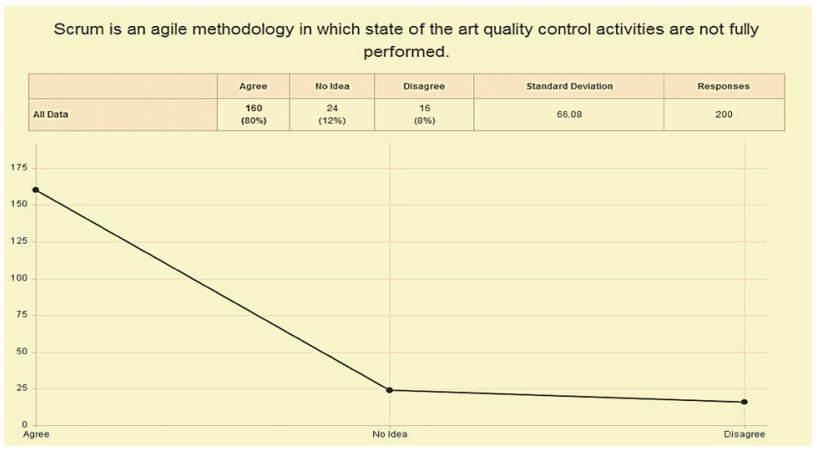

Figure 6. Scrum and quality control activities.

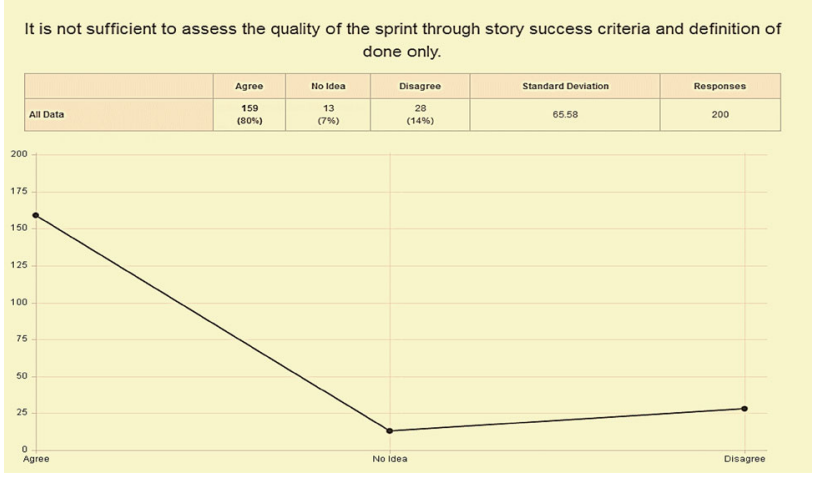

Figure 7. State of quality of the sprint through story success criteria and definition-of-done.

and 111 developers and testers among the survey participants. It has been observed that $94.5 \%$ IT professionals claimed that scrum was fully implemented in their organizations. Since all of the survey participants were IT professionals having sound knowledge regarding scrum, the issues and challenges, we can articulate that we have selected right candidates for our survey. Further, 39\% of the participants strongly agreed and $35 \%$ of the participants agreed with the assertion that scrum was easy for adoption but difficult to manage. Survey results show that $31 \%$ professionals strongly agreed and $50 \%$ agreed that state of the art QC activities were not being performed in scrum due to the short cycle of sprint. Moreover, 39\% professionals strongly agreed and $44.5 \%$ agreed that it was not adequate to validate the sprint with only story success criteria. In this survey, we also focussed on the validation of our proposed enhanced solution to incorporate QC activities in scrum and asses the quality of the product being developed using frequency of leftover functional bugs. Survey results show that $26.5 \%$ professionals strongly agreed and $64.5 \%$ agree on enhancement of current scrum for incorporation of QC activities. Furthermore, $40 \%$ professionals strongly agreed and $48.5 \%$ agreed on incorporation of test backlog to maintain the 


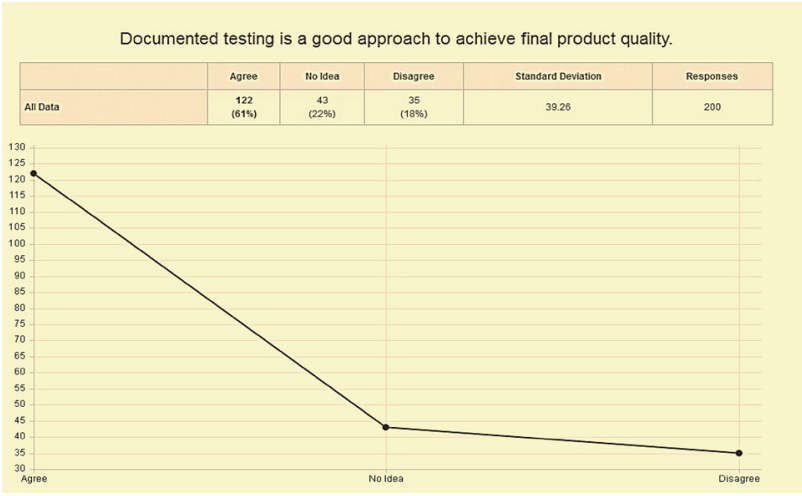

Figure 8. Achieving product quality through documented testing.

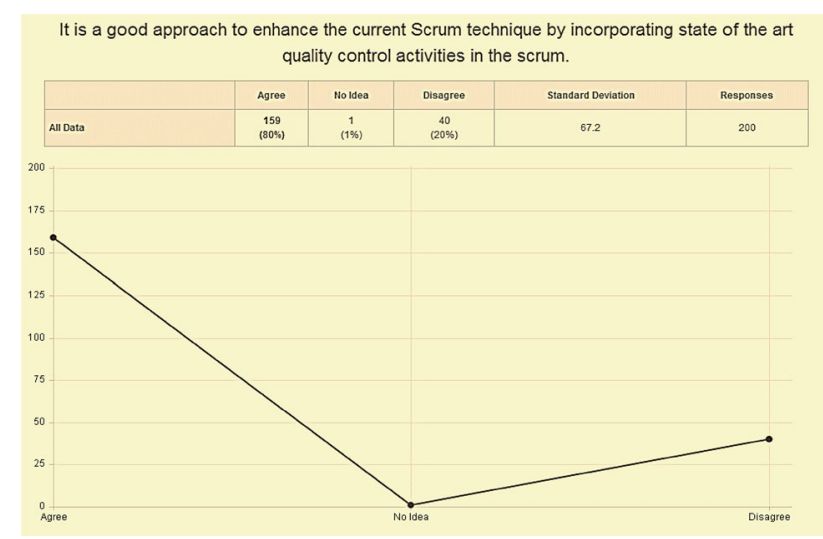

Figure 9. Enhancing scrum through state-of-the-art quality control activities.

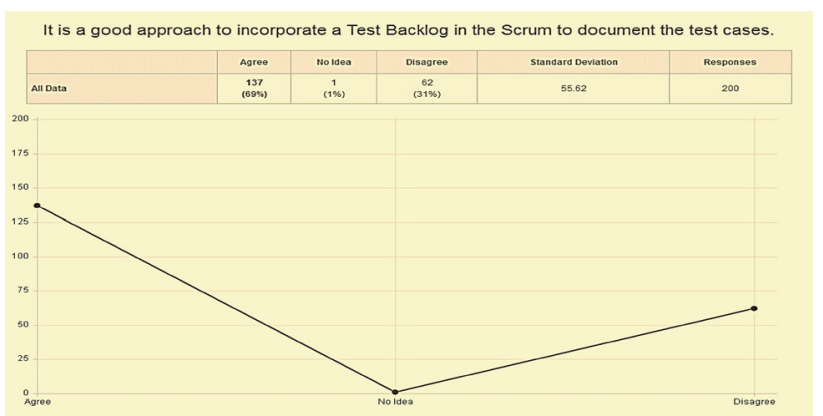

Figure 10. Incorporating test backlog in the scrum to document the test cases.

test cases against user stories in sprint cycle. Likewise, $57 \%$ professionals strongly agreed and 39\% agreed that QC teams should work in parallel with the development team in scrum. In addition, $32.2 \%$ professionals strongly agreed and $63.3 \%$ agreed that quality of product under development could be assessed through number of functional bugs. Finally, $43.2 \%$ professionals strongly agreed

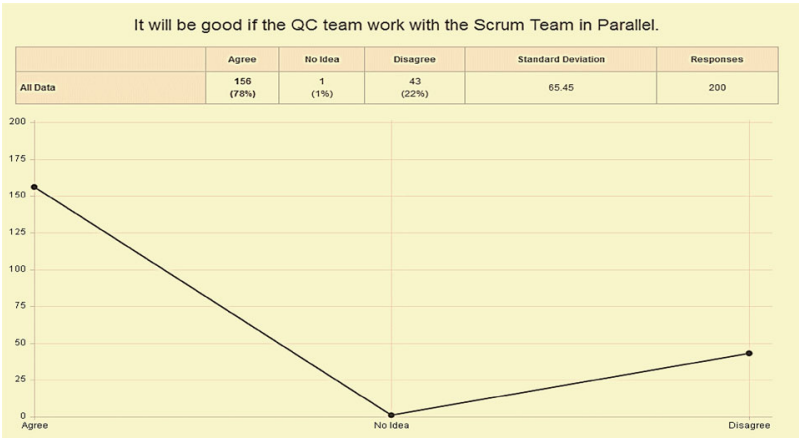

Figure 11. QC and scrum teams working in parallel.

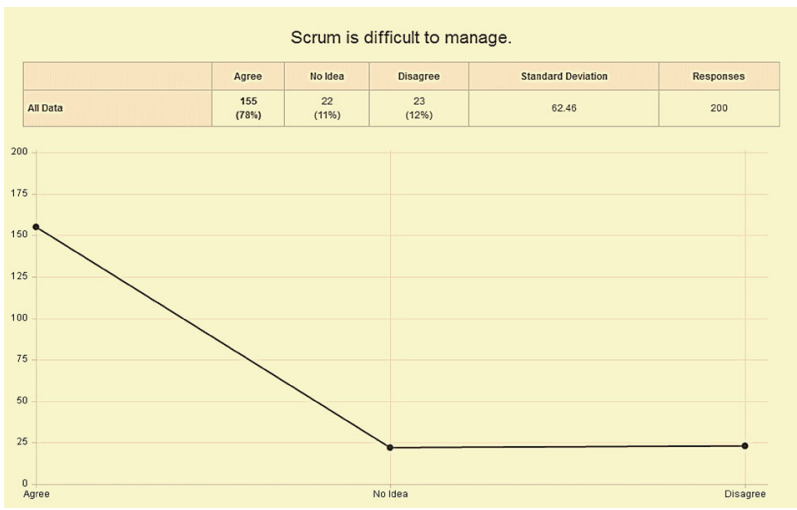

Figure 12. Complexity of scrum.

and $51.3 \%$ agreed that the quality of product goes down if the number of functional bugs increases with the passage of time. Overall, the survey results were in conformance with our proposed model and helped us validate the proposed solution.

\section{Conclusion and future work}

In this paper we have addressed the issue of adding QC measures in scrum, in particular, by dealing with the concept of test backlog. The amplified scrum model proposed by us provides quality assessment based on functional bugs frequency. We proposed a set of testing activities in much the same way as a sprint is conducted. These activities are based on the proposed test backlog concept, which is a set of test cases. We validated our approach using a case study and also performed a survey about QC and other aspects of scrum.

In scrum, most of the organizations ignore QC activities, especially test documentation, or adopt it at a very abstract level such as performing sanity and acceptance testing. We ascertain that only sanity and acceptance testing is not sufficient to develop a quality product. Other QC activities like test case planning, 
documentation and execution should also be performed. For this purpose, we proposed an enhanced scrum model to implement state of the art QC activities and assess quality of the product in scrum. The concept of test backlog is also introduced in this paper. The test backlog contains the test cases. The QC process starts as the sprint starts and the test cases are designed on the basis of user stories contained in the sprint. The quality assessment is calculated on the basis of frequency of leftover functional bugs. This research serves as a starting point for researchers and scrum practitioners and is likely to augment research in the area of scrum and software QC. There are some challenges observed in this research, which need further investigation. The first challenge is making a scrum tool that encapsulates the concept of test backlog and bug tracking through which we can assess quality of the product. Further, the technique should be applied to large-sized projects. To achieve better quality assessment, extensive statistical analysis is required.

\section{References}

[1] Schwaber K and Sutherland J 2011 The scrum guide. Scrum Alliance, Indianapolis IN, USA

[2] Larusdottir M K, Bjarnadottir E R and Gulliksen J 2010 The focus on usability in testing practices in industry. In: Humancomputer interaction, pp. 98-109. Springer BerlinHeidelberg

[3] Green R, Mazzuchi T and Sarkani S 2010 Understanding the role of synchronous \& asynchronous communication in agile software development and its effects on quality. J. Inf. Technol. Manage. 21(2): 8

[4] Löffler R, Güldali B and Geisen S 2010 Towards modelbased acceptance testing for scrum. Softwaretechnik-Trends, GI, Zürich

[5] Sabir F, Ullah A, Khan M A, Rafique M Q and Ali M 2012 Improved scrum model with SDLC. J. Basic Appl. Sci. Res. 2(7): 6647-6656

[6] Sutherland J and Schwaber K 2007 The scrum papers: nuts, bolts, and origins of an agile method. Scrum, Inc., Boston

[7] Vlaanderen K, Jansen S, Brinkkemper S and Jaspers E 2011 The agile requirements refinery: applying SCRUM principles to software product management. Inf. Softw. Technol. 53(1): $58-70$

[8] Scharff C and Verma R 2010 Scrum to support mobile application development projects in a just-in-time learning context. In: Proceedings of the 2010 ICSE Workshop on Cooperative and Human Aspects of Software Engineering, pp. 25-31. ACM, Cape Town, South Africa
[9] Akif R and Majeed H 2012 Issues and challenges in scrum implementation. Int. J. Sci. Eng. Res. 3(8): 1-4

[10] Vlietland J and van Vliet H 2015 Towards a governance framework for chains of Scrum teams. Inf. Softw. Technol. 57: 52-65

[11] Paulk M 2014 On empirical research into scrum adoption. Carnegie Mellon University, Pittsburgh, PA: Institute for Software Research

[12] Stettina C J and Hörz J 2015 Agile portfolio management: an empirical perspective on the practice in use. Int. J. Proj. Manage. 33(1): 140-152

[13] Rodriguez G, Soria Á and Campo M 2015 Virtual Scrum: a teaching aid to introduce undergraduate software engineering students to scrum. Comput. Appl. Eng. Educ. 23(1): 147-156

[14] von Wangenheim C G, Savi R and Borgatto A F 2013 SCRUMIA - an educational game for teaching SCRUM in computing courses. J. Syst. Softw. 86(10): 2675-2687

[15] Hongying G and Cheng Y 2011 A customizable agile software Quality Assurance model. In: Proceedings of the 5th International Conference on New Trends in Information Science and Service Science (NISS), vol. 2, pp. 382-387. IEEE, Macao, China

[16] Li J, Moe N B and Dybå T 2010 Transition from a plandriven process to scrum: a longitudinal case study on software quality. In: Proceedings of the 2010 ACM-IEEE International Symposium on Empirical Software Engineering and Measurement, p. 13. ACM, Bolzano, Italy

[17] Bhasin S 2012 Quality assurance in agile: a study towards achieving excellence. In: Proceedings of AGILE India (AGILE INDIA), pp. 64-67. IEEE, Bengaluru, India

[18] Parvez M and Parvaz M 2012 An efficient approach to interact between agile scrum and quality management system. Adv. Inf. Technol. Manage. 1(3): 105-110

[19] Häger F, Kowark T, Krüger J, Vetterli C, Übernickel F and Uflacker M 2015 DT@ Scrum: integrating design thinking with software development processes. In: Design thinking research, pp. 263-289. Springer, Cham, Switzerland

[20] Tomanek M, Cermak R and Smutny Z 2015 A conceptual framework for web development projects based on project management and agile development principles. arXiv preprint arXiv: 1502.04297

[21] Kaur C and Kumar V 2015 Product backlog prioritization in scrum: a review. Int. J. Mod. Comput. Sci. 3(2): 21-24

[22] Glória I, Oliveira R and Chaves M 2014 A Proposal for using Web 2.0 technologies in SCRUM. In: ECIS 2014 Proceedings

[23] Usman M, Soomro T R and Brohi M N 2014 Embedding project management into XP, SCRUM and RUP. Eur. Sci. J. 10(15): 293-307

[24] Stålhane T, Myklebust T and Hanssen G K 2012 The application of Safe Scrum to IEC 61508 certifiable software. In: 11th International Probabilistic Safety Assessment and Management Conference and the Annual European Safety and Reliability Conference, pp. 6052-6061. Helsinki, Finland 